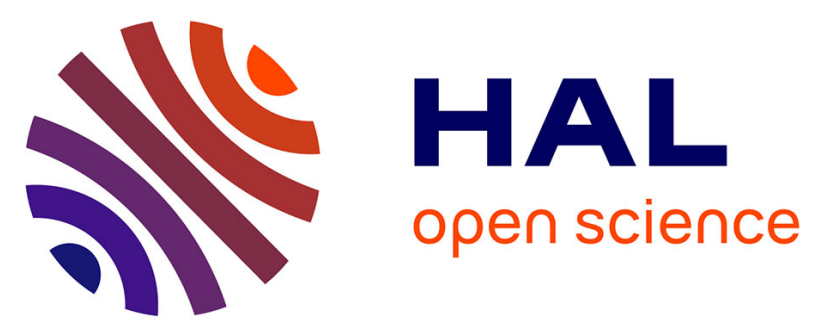

\title{
Etude anatomique du thalamus en IRM 4,7 Tesla et apport pour la segmentation manuelle des noyaux intrathalamiques en chirurgie stéréotaxique
}

Jean-Jacques Lemaire, Vivien Mendes Martins, Laurent Sakka, François

Vassal, François Caire, Jérôme Coste, Toufic Khalil, Jean J Chazal

\section{To cite this version:}

Jean-Jacques Lemaire, Vivien Mendes Martins, Laurent Sakka, François Vassal, François Caire, et al.. Etude anatomique du thalamus en IRM 4,7 Tesla et apport pour la segmentation manuelle des noyaux intrathalamiques en chirurgie stéréotaxique. Réunion de la Société de Neurochirurgie de Langue Française, Société de Neurochirurgie de Langue Française, Nov 2007, Paris, France. pp.419, 10.1016/j.neuchi.2007.09.018 . hal-01899229

\section{HAL Id: hal-01899229 \\ https://hal.science/hal-01899229}

Submitted on 8 Nov 2018

HAL is a multi-disciplinary open access archive for the deposit and dissemination of scientific research documents, whether they are published or not. The documents may come from teaching and research institutions in France or abroad, or from public or private research centers.
L'archive ouverte pluridisciplinaire HAL, est destinée au dépôt et à la diffusion de documents scientifiques de niveau recherche, publiés ou non, émanant des établissements d'enseignement et de recherche français ou étrangers, des laboratoires publics ou privés. 


\title{
Etude anatomique du thalamus en IRM 4,7 Tesla et apport pour la segmentation manuelle des noyaux intrathalamiques en chirurgie stéréotaxique
}

\author{
Lemaire Jean-Jacques, Mendes Martins Vivien, Sakka Laurent, Vassal François, \\ Caire François, Coste Jérôme, Khalil Toufic, Chazal Jean
}

Service de Neurochirurgie A, Hôpital Gabriel-Montpied, CHU, Clermont-Ferrand, France ; Inserm, ERI 14, Clermont-Ferrand, France.

Introduction. La segmentation manuelle du thalamus sur l'imagerie par résonance magnétique nucléaire (IRM) en clinique à 1,5 Tesla reste difficile. Nous avons réalisé une étude anatomique morphologique à 4,7 Tesla du thalamus pour revisiter sa systématisation et utiliser cette information pour l'identification (segmentation) des noyaux en routine clinique.

Matériel - Méthode. Deux cent cinquante-six coupes centrées sur les ganglions de la base d'un spécimen anatomique ont servis pour l'étude morphologique (voxel $=0,253$ $\mathrm{mm}^{3}$ ). La segmentation intra-thalamique a été réalisée manuellement en fonction des contrastes spontanés et de la position relative des structures. Les connaissances issues d'atlas stéréotaxiques et de livres d'anatomie ont servi à l'identification primaire qui a été finalisée grâce au suivi tridimensionnel.

Résultats ou Cas rapporté. L'ensemble des noyaux intra-thalamiques a pu être segmenté : la région ventro-latérale, centrale et dorsale, le thalamus postérieur et médian, ainsi que des structures liées (zona incerta, substance grillagée d'Arnold, champs de Forel). L'information 3D infra-millimétrique a permis une meilleure compréhension de la systématisation, directement exploitable, car les contrastes sont identiques, pour la reconnaissance des noyaux en conditions cliniques en IRM dès 1,5 Tesla.

Conclusion. Ce travail a permis de revisiter l'anatomie complexe du thalamus. II a été appliqué pour la cartographie morphologique IRM chez 12 patients. La segmentation (son principe et ses résultats) et l'application clinique sont discutées. 